\title{
Existence of Standing Waves for a Generalized Davey-Stewartson System
}

\author{
Xiaoxiao Hu, ${ }^{1}$ Xiao-xun Zhou, ${ }^{2}$ Wu Tunhua, ${ }^{1}$ and Min-Bo Yang ${ }^{3}$ \\ ${ }^{1}$ School of Information and Engineering, Wenzhou Medical College, Wenzhou, Zhejiang 325035, China \\ ${ }^{2}$ School of Marxism, Tongji University, Shanghai 200092, China \\ ${ }^{3}$ Department of Mathematics, Zhejiang Normal University, Jinhua, Zhejiang 321004, China
}

Correspondence should be addressed to Xiaoxiao Hu; smallapple12345@163.com

Received 13 September 2012; Revised 26 December 2012; Accepted 14 January 2013

Academic Editor: Norimichi Hirano

Copyright (C) 2013 Xiaoxiao Hu et al. This is an open access article distributed under the Creative Commons Attribution License, which permits unrestricted use, distribution, and reproduction in any medium, provided the original work is properly cited.

\begin{abstract}
The purpose of this paper is to investigate the existence of standing waves for a generalized Davey-Stewartson system. By reducing the system to a single Schrödinger equation problem, we are able to establish some existence results for the system by variational methods.
\end{abstract}

\section{Introduction and Main Results}

In this paper, we are going to consider the existence of standing waves for a generalized Davey-Stewartson system in $\mathbb{R}^{3}$

$$
\begin{aligned}
i \psi_{t}+\Delta \psi & =b(x) \psi \varphi_{x_{1}}-a(x)|\psi|^{p-2} \psi, \\
-\Delta \varphi & =\left(b(x)|\psi|^{2}\right)_{x_{1}} .
\end{aligned}
$$

Here $\Delta$ is the Laplacian operator in $\mathbb{R}^{3}$ and $i$ is the imaginary unit, $a(x), b(x)$, and $p$ satisfy some additional assumptions.

The Davey-Stewartson system is a model for the evolution of weakly nonlinear packets of water waves that travel predominantly in one direction, but in which the amplitude of waves is modulated in two spatial directions. They are given as

$$
\begin{aligned}
i \psi_{t}+\psi_{x x}+\delta \psi_{y y} & =b_{1} \psi \varphi_{x}-a|\psi|^{2} \psi, \\
m \varphi_{x x}+\varphi_{y y} & =b_{2}\left(|\psi|^{2}\right)_{x},
\end{aligned}
$$

where $a, b_{1}, b_{2} \in \mathbb{R}, \psi(t, x, y)$ is the complex amplitude of the shortwave and $\varphi(t, x, y)$ is the real longwave amplitude [1]. The physical parameters $\delta$ and $m$ play a determining role in the classification of this system. Depending on their signs, the system is elliptic-elliptic, elliptic-hyperbolic, hyperbolicelliptic, and hyperbolic-hyperbolic [2], although the last case does not seem to occur in the context of water waves.
As we know, the system can be reduced to a single Schrödinger equation by using Fourier transforms. Indeed, let $E_{1}$ be the singular integral operator defined by

$$
\mathscr{F}\left\{E_{1}(\psi)\right\}(\xi)=\sigma_{1}(\xi) \mathscr{F}(\psi)(\xi),
$$

where $\sigma_{1}(\xi)=\xi_{1}^{2} /|\xi|^{2}, \xi \in \mathbb{R}^{3}$, and $\mathscr{F}$ denotes the Fourier transform:

$$
\mathscr{F}(\psi)(\xi)=\left(\frac{1}{2 \pi}\right)^{3 / 2} \int e^{-i \xi x} \psi(x) d x .
$$

Then the generalized Davey-Stewartson system can be reduced to the following single nonlocal Schrödinger equation

$$
-i \psi_{t}-\Delta \psi=a(x)|\psi|^{p-2} \psi+b(x) E_{1}\left(b(x)|\psi|^{2}\right) \psi .
$$

In this paper, we are interested in the existence of standing waves for the above equation, that is, solutions in the form of

$$
\begin{gathered}
\psi(t, x)=e^{i \omega t} \phi(x), \\
\varphi(t, x)=v(x),
\end{gathered}
$$

where $\omega>0, \phi, v \in H^{1}\left(\mathbb{R}^{3}\right)$. Then if $(\psi, \varphi)$ is a solution of (1), then we can see that $\phi$ must satisfy the following Schrödinger problem:

$$
-\Delta \phi+\omega \phi=b(x) E_{1}\left(b(x)|\phi|^{2}\right) \phi+a(x)|\phi|^{p-2} \phi .
$$


We will consider the generalized Davey-Stewartson system with perturbation. Under suitable assumptions on the coefficients $a(x), b(x)$, the problem can be viewed as the perturbation of the generalized Davey-Stewartson system considered in $[2,3]$. Here we will not use the critical point theory or the minimizing methods to establish the existence results. Moreover, we will not use Lion's Concentrationcompactness principle to overcome the difficulty of losing compactness. Instead, we will apply the perturbation method developed by Ambrosetti and Badiale in $[4,5]$ to show the existence of solutions of (8) and (9). In [4, 5], Ambrosetti and Badiale established an abstract theory to reduce a class of perturbation problems to a finite dimensional one by some careful observation on the unperturbed problems and the Lyapunov-Schmit reduction procedure. This method has also been successfully applied to many different problems, see [6] for examples. In this paper we are going to consider the following two types of perturbed problems for generalized Davey-Stewartson system. Consider

$$
\begin{aligned}
& -\Delta \phi+\phi=\varepsilon b(x) E_{1}\left(b(x)|\phi|^{2}\right) \phi+(1+\varepsilon a(x))|\phi|^{p-2} \phi \\
& -\Delta \phi+\omega \phi=b(x) E_{1}\left(b(x)|\phi|^{2}\right) \phi+a(x)|\phi|^{p-2} \phi
\end{aligned}
$$

The main results of the paper are the following theorems.

Theorem 1. Assume that $2<p<6, a(x) \in L^{6 /(6-p)}\left(\mathbb{R}^{3}\right)$ and $b(x) \in L^{6}\left(\mathbb{R}^{3}\right)$. Take the function $U$ from Proposition 4 in Section 2, if there holds

$$
\frac{1}{4} \int b(x) E_{1}\left(b(x)|U|^{2}\right)|U|^{2}+\frac{1}{p} \int a(x)|U|^{p} \neq 0,
$$

then for any $\varepsilon$ small, there exists at least one solution of problem (8).

Theorem 2. Let $\omega=\varepsilon^{2}$, suppose $2<p<4$, and there exists a positive constant $A$ such that $a(x), b(x)$ satisfy

$\left(a_{1}\right) a(x)-A$ is continuous, bounded and $a(x)-A \in L^{1}\left(\mathbb{R}^{3}\right)$ with $\int(a(x)-A) \neq 0$;

$\left(b_{1}\right) b(x)$ is continuous, bounded and $b \in L^{2}\left(\mathbb{R}^{3}\right)$.

Then for $\varepsilon>0$ small enough, there exists a solution $\phi_{\varepsilon}$ in $H^{1}\left(\mathbb{R}^{3}\right)$ for problem (9). Moreover, if $2<p<2+4 / 3$, then $\phi_{\varepsilon} \rightarrow 0$ as $\varepsilon \rightarrow 0$.

Theorem 3. Let $\omega=\varepsilon^{2}$, suppose $2<p<4$ and $a(x), b(x)$ satisfy $\left(b_{1}\right)$ and

$\left(a_{2}\right) a-A$ is continuous and there exist $L \neq 0$ and $0<\gamma<3$ such that $|x|^{\gamma}(a(x)-A) \rightarrow L$ as $|x| \rightarrow \infty$.

Then for $\varepsilon>0$ small enough, there exists a solution $\phi_{\varepsilon}$ in $H^{1}\left(\mathbb{R}^{3}\right)$ for problem (9). Moreover, if $2<p<2+4 / 3$, then $\phi_{\varepsilon} \rightarrow 0$ as $\varepsilon \rightarrow 0$.

Throughout this paper, we denote the norm of $H^{1}\left(\mathbb{R}^{3}\right)$ by

$$
\|u\|=\left(\int|\nabla u|^{2}+u^{2}\right)^{1 / 2}
$$

and by $|\cdot|_{s}$ we denote the usual $L^{s}$-norm; $C, C_{i}$ stand for different positive constants.

The paper is organized as follows. In Section 2, we outline the abstract critical point theory for perturbed functionals and give some properties for the singular operator $E_{1}$. In Section 3, we prove the main results by some lemmas.

\section{The Abstract Theorem}

To prove the main results, we need the following known propositions.

Proposition 4. For any positive constant $A$, consider the following problem, $2<p<2^{*}$ :

$$
\begin{gathered}
-\Delta u+u=A|u|^{p-2} u, \\
u>0, \quad u \in H^{1}\left(\mathbb{R}^{3}\right) .
\end{gathered}
$$

There is a unique positive radial solution $U$, which satisfies the following decay property:

$$
\lim _{r \rightarrow \infty} U(r) r e^{r}=C>0, \quad \lim _{r \rightarrow \infty} \frac{U^{\prime}(r)}{U(r)}=-1, \quad r=|x|,
$$

where $C>0$ is a constant. The function $U$ is a critical point of $C^{2}$ functional $I_{0}: H^{1}\left(\mathbb{R}^{3}\right) \rightarrow \mathbb{R}$ defined by

$$
I_{0}(u)=\frac{1}{2}\|u\|^{2}-\frac{A}{p} \int_{\mathbb{R}^{3}}|u|^{p} .
$$

Moreover, $I_{0}$ possesses a 3-dimensional manifold of critical points

$$
Z=\left\{z_{\theta}=U(x+\theta), \theta \in \mathbb{R}^{3}\right\}
$$

Set

$$
Q(u):=I_{0}^{\prime \prime}(U)[u, u]=\int_{\mathbb{R}^{3}}\left[|\nabla u|^{2}+u^{2}-p A U^{p-2} u^{2}\right]
$$

and denote $X=\operatorname{span}\left\{\partial U / \partial x_{i}, 1 \leq i \leq 3\right\}$. We have

(1) $Q(U)=(2-p) A \int_{\mathbb{R}^{3}} U^{p} d x<0$,

(2) $\operatorname{Ker} Q=X$,

(3) $Q(w) \geq C\|w\|^{2}$, for all $w \in(\mathbb{R} U \oplus X)^{\perp}$.

In the following, we outline the abstract theorem of a variational method to study critical points of perturbed functionals. Let $E$ be a real Hilbert space, we will consider the perturbed functional defined on it of the form

$$
I_{\varepsilon}(u)=I_{0}(u)+G(\varepsilon, u)
$$

where $I_{0}: E \rightarrow \mathbb{R}$ and $G: \mathbb{R} \times E \rightarrow \mathbb{R}$. We need the following hypotheses and assume that

(1) $I_{0}$ and $G$ are $C^{2}$ with respect to $u$;

(2) $G$ is continuous in $(\varepsilon, u)$ and $G(0, u)=0$ for all $u$; 
(3) $G^{\prime}(\varepsilon, u)$ and $G^{\prime \prime}(\varepsilon, u)$ are continuous maps from $\mathbb{R} \times$ $E \rightarrow E$ and $L(E, E)$, respectively, and $L(E, E)$ is the space of linear continuous operators from $E$ to $E$.

(4) There is a $d$-dimensional $C^{2}$ manifold $Z, d \geq 1$, consisting of critical points of $I_{0}$, and such a $Z$ will be called a critical manifold of $I_{0}$.

(5) let $T_{\theta} Z$ denote the tangent space to $Z$ at $z_{\theta}$, the manifold $Z$ is nondegenerate in the following sense:

$\operatorname{Ker}\left(I_{0}^{\prime \prime}(z)\right)=T_{\theta} Z$ and $I_{0}^{\prime \prime}\left(z_{\theta}\right)$ is an index-0 Fredholm operator for any $z_{\theta} \in Z$.

(6) There exists $\alpha>0$ and a continuous function $\Gamma: Z \rightarrow$ $\mathbb{R}$ such that

$$
\begin{aligned}
& \Gamma(z)=\lim _{\varepsilon \rightarrow 0} \frac{G(\varepsilon, z)}{\varepsilon^{\alpha}}, \\
& G^{\prime}(\varepsilon, z)=o\left(\varepsilon^{\alpha / 2}\right) .
\end{aligned}
$$

Consider the existence of critical points of the perturbed problem

$$
I_{\varepsilon}^{\prime}(u)=0
$$

We want to look for solutions of the form $u=z+w$ with $z \in Z$ and $w \in W=\left(T_{\theta} Z\right)^{\perp}$. Then we can reduce the problem to a finite-dimensional one by Lyapunov-Schmit procedure, that is, it is equivalent to solve the following system:

$$
\begin{gathered}
P I_{\varepsilon}^{\prime}(z+w)=0, \\
(I-P) I_{\varepsilon}^{\prime}(z+w)=0 .
\end{gathered}
$$

Here $P$ is the orthogonal projection onto $W$. Under the conditions above, the first equation in this system can be solved by implicit function theorem, and then by using the Taylor expansion, we obtain for $u=z+w(\varepsilon, z)$

$$
I_{\varepsilon}(u)=I_{0}(z)+\varepsilon^{\alpha} \Gamma(z)+o\left(\varepsilon^{\alpha}\right) .
$$

In $[4,5]$ the following abstract theorem is proved.

Lemma 5. Suppose assumptions (1)-(6) are satisfied, and there exists $\delta>0$ and $z^{*} \in Z$ such that

$$
\text { either } \min _{\left\|z-z^{*}\right\|=\delta} \Gamma(z)>\Gamma\left(z^{*}\right) \quad \text { or } \max _{\left\|z-z^{*}\right\|=\delta} \Gamma(z)<\Gamma\left(z^{*}\right) \text {. }
$$

Then for any $\varepsilon$ small, there exists $u_{\varepsilon}$ which is a critical point of $I_{\varepsilon}$.

We give some facts about the singular integral $E_{1}$ in Cipolatti [2].

Lemma 6. Let $E_{1}$ be the singular integral operator defined in Fourier variable by

$$
\mathscr{F}\left\{E_{1}(\psi)\right\}(\xi)=\sigma_{1}(\xi) \mathscr{F}(\psi)(\xi),
$$

where $\sigma_{1}(\xi)=\xi_{1}^{2} /|\xi|^{2}, \xi \in \mathbb{R}^{3}$, and $\mathscr{F}$ denotes the Fourier transform:

$$
\mathscr{F}(\psi)(\xi)=\left(\frac{1}{2 \pi}\right)^{3 / 2} \int e^{-i \xi x} \psi(x) d x .
$$

For $1<p<\infty, E_{1}$ satisfies the following properties:

(1) $E_{1} \in \mathscr{L}\left(L^{p}, L^{p}\right)$.

(2) if $\psi \in H^{1}\left(\mathbb{R}^{3}\right)$, then $E_{1}(\psi) \in H^{1}\left(\mathbb{R}^{3}\right)$.

(3) $E_{1}$ preserves the following operations:

$$
\begin{aligned}
& \text { translation: } E_{1}(\psi(\cdot+y))(x)=E_{1}(\psi)(x+y), y \in \\
& \mathbb{R}^{3} \text {. } \\
& \text { dilation: } E_{1}(\psi(\lambda \cdot))(x)=E_{1}(\psi)(\lambda x), \lambda>0 . \\
& \text { conjugation: } \overline{E_{1}(\psi)}=E_{1}(\bar{\psi}), \bar{\psi} \text { is the complex } \\
& \text { conjugate of } \psi .
\end{aligned}
$$

\section{Proof of the Main Results}

In this section, we would apply the abstract tools of the previous section to prove the main results. First let us consider (8), the corresponding energy functional $I_{\varepsilon}: H^{1}\left(\mathbb{R}^{3}\right) \rightarrow \mathbb{R}$ can be defined as

$$
\begin{aligned}
I_{\varepsilon}(\phi)= & \frac{1}{2}\|\phi\|^{2} \\
& -\frac{\varepsilon}{4} \int b(x) E_{1}\left(b(x)|\phi|^{2}\right)|\phi|^{2} \\
& -\frac{1}{p} \int(1+\varepsilon a(x))|\phi|^{p} .
\end{aligned}
$$

It is easy to see that $I_{\varepsilon}: H^{1}\left(\mathbb{R}^{3}\right) \rightarrow \mathbb{R}$ is of $C^{2}$, and thus $\phi$ is a solution of (8) if and only if $\phi$ is a critical point of the action functional $I_{\varepsilon}(\phi)$.

Proof of Theorem 1. Set

$$
\begin{gathered}
I_{0}(\phi)=\frac{1}{2}\|\phi\|^{2}-\frac{1}{p} \int|\phi|^{p}, \\
G(\phi)=-\frac{1}{p} \int a(x)|\phi|^{p}-\frac{1}{4} \int b(x) E_{1}\left(b(x)|\phi|^{2}\right)|\phi|^{2},
\end{gathered}
$$

then $I_{\varepsilon}(u)$ can be rewritten as

$$
I_{\varepsilon}(\phi)=I_{0}(\phi)+\varepsilon G(\phi) .
$$

Thus $I_{0}(\phi)$ and $G(\phi)$ are both $C^{2}$ with respect to $\phi$. To apply Lemma 5, by Proposition 4, we need only to check that

$$
\begin{gathered}
\lim _{|\theta| \rightarrow \infty} \Gamma(\theta)=0, \\
\Gamma(\theta):=\left.G\right|_{Z}=-\frac{1}{p} \int a(x)\left|z_{\theta}\right|^{p} \\
-\frac{1}{4} \int b(x) E_{1}\left(b(x)\left|z_{\theta}\right|^{2}\right)\left|z_{\theta}\right|^{2} .
\end{gathered}
$$


From the fact that $a \in L^{6 /(6-p)}\left(\mathbb{R}^{3}\right)$, for any $T>0$, we have

$$
\begin{aligned}
\left.\left|\int a(x)\right| z_{\theta}\right|^{p} \mid \leq & \left.\left|\int_{|x| \leq T} a(x)\right| z_{\theta}\right|^{p}|+| \int_{|x| \geq T} a(x)\left|z_{\theta}\right|^{p} \mid \\
\leq & \left(\int_{|x| \leq T}|a(x)|^{6 /(6-p)}\right)^{(6-p) / 6} \\
& \times\left(\int_{|x| \leq T}\left|z_{\theta}\right|^{6}\right)^{p / 2^{*}} \\
& +\left(\int_{|x| \geq T}|a(x)|^{6 /(6-p)}\right)^{(6-p) / 6} \\
& \times\left(\int_{|x| \geq T}\left|z_{\theta}\right|^{6}\right)^{p / 6} .
\end{aligned}
$$

Since $U$ exponentially decays at infinity, we know the right side of the equality goes to 0 , if $\theta \rightarrow \infty$.

Let $B_{1}$ be the quadratic functional on $L^{2}$ defined by

$$
B_{1}(\phi)=\int \sigma_{1}(\xi)|\mathscr{F}(b \cdot \phi)(\xi)|^{2} d \xi
$$

it follows from the Parseval identity that

$$
B_{1}(\phi)=\int E_{1}(b \cdot \phi) \overline{b \cdot \phi}
$$

and in particular we have

$$
0<B_{1}(\phi) \leq|b(x) \phi|_{2}^{2} .
$$

Then for any $T>0$, we have

$$
\begin{aligned}
\mid \int b(x) & E_{1}\left(b(x)\left|z_{\theta}\right|^{2}\right)\left|z_{\theta}\right|^{2} \mid \\
\leq & \left.\left.\int|b(x)| z_{\theta}\right|^{2}\right|^{2} \\
\leq & \left(\int_{|x| \leq T}|b(x)|^{6}\right)^{1 / 3}\left(\int_{|x| \leq T}\left|z_{\theta}\right|^{6}\right)^{2 / 3} \\
& +\left(\int_{|x| \geq T}|b(x)|^{6}\right)^{1 / 3}\left(\int_{|x| \geq T}\left|z_{\theta}\right|^{6}\right)^{2 / 3} .
\end{aligned}
$$

Since $U$ exponentially decays at infinity, the right side of the inequality (32) goes to 0 . Thus from (29) and (32) above we soon get

$$
\lim _{|\theta| \rightarrow \infty} \Gamma(\theta)=0
$$

Then by assumption (10) that

$$
\frac{1}{4} \int b(x) E_{1}\left(b(x)|U|^{2}\right)|U|^{2}+\frac{1}{p} \int a(x)|U|^{p} \neq 0,
$$

we know $\Gamma(0) \neq 0$. Thus, the conclusion follows from Lemma 5 that any strict maximum or minimum of $\Gamma$ gives rise to a critical point of the perturbed functional and hence to a solution of $(8)$.
We are going to consider problem (9). Set

$$
\begin{gathered}
\omega=\varepsilon^{2}, \\
\phi(x)=\varepsilon^{2 /(p-2)} u(\varepsilon x) .
\end{gathered}
$$

We have

$$
\begin{aligned}
-\Delta u+u= & a\left(\frac{x}{\varepsilon}\right)|u|^{p-2} u \\
& +\varepsilon^{2(4-p) /(p-2)} b\left(\frac{x}{\varepsilon}\right) \\
& \times E_{1}\left(b\left(\frac{x}{\varepsilon}\right)|u|^{2}\right) u .
\end{aligned}
$$

It can be proved that $\phi(x)=\varepsilon^{2 /(p-2)} u(\varepsilon x) \in H^{1}\left(\mathbb{R}^{3}\right)$ is a solution of system (9) if and only if $u \in H^{1}\left(\mathbb{R}^{3}\right)$ is a critical point of the functional $I_{\varepsilon}: H^{1}\left(\mathbb{R}^{3}\right) \rightarrow \mathbb{R}$ defined by

$$
\begin{aligned}
I_{\varepsilon}(u)= & \frac{1}{2}\|u\|^{2}-\frac{1}{p} \int a\left(\frac{x}{\varepsilon}\right)|u|^{p} \\
& -\frac{\varepsilon^{2(4-p) /(p-2)}}{4} \\
& \times \int b\left(\frac{x}{\varepsilon}\right) E_{1}\left(b\left(\frac{x}{\varepsilon}\right)|u|^{2}\right)|u|^{2} .
\end{aligned}
$$

Set

$$
I_{0}(u)=\frac{1}{2}\|u\|^{2}-\frac{A}{p} \int|u|^{p} .
$$

Then $I_{\varepsilon}(u)$ can be rewritten as

$$
\begin{aligned}
I_{\varepsilon}(u)= & I_{0}(u)+\frac{1}{p} \int\left(A-a\left(\frac{x}{\varepsilon}\right)\right)|u|^{p} \\
& -\frac{\varepsilon^{2(4-p) /(p-2)}}{4} \\
& \times \int b\left(\frac{x}{\varepsilon}\right) E_{1}\left(b\left(\frac{x}{\varepsilon}\right)|u|^{2}\right)|u|^{2} .
\end{aligned}
$$

Define

$$
\begin{aligned}
\widetilde{G}(\varepsilon, u)= & \frac{1}{p} \int\left(A-a\left(\frac{x}{\varepsilon}\right)\right)|u|^{p} \\
& -\frac{\varepsilon^{2(4-p) /(p-2)}}{4} \int b\left(\frac{x}{\varepsilon}\right) E_{1}\left(b\left(\frac{x}{\varepsilon}\right)|u|^{2}\right)|u|^{2} \\
= & \widetilde{G}_{1}(\varepsilon, u)+\widetilde{G}_{2}(\varepsilon, u)
\end{aligned}
$$

and for $i=1,2$

$$
G_{i}(\varepsilon, u)= \begin{cases}\widetilde{G}_{i}(\varepsilon, u), & \text { if } \varepsilon \neq 0 \\ 0, & \text { if } \varepsilon=0\end{cases}
$$

Lemma 7. Under assumptions $\left(a_{1}\right)$ and $\left(b_{1}\right), G=G_{1}+G_{2}$ is continuous in $(\varepsilon, u)$. 
Proof. From the proof of Lemma 4.1 in [7], we know $G_{1}$ is continuous in $(\varepsilon, u) \in \mathbb{R} \times H^{1}\left(\mathbb{R}^{3}\right)$, and hence we only need to prove that $G_{2}$ is continuous in $(\varepsilon, u)$.

If $(\varepsilon, u) \rightarrow\left(\varepsilon_{0}, u_{0}\right)$, with $\varepsilon_{0} \neq 0$. Then we can estimate that

$$
\begin{aligned}
& 4\left|G_{2}(\varepsilon, u)-G_{2}\left(\varepsilon_{0}, u_{0}\right)\right| \\
& =\left.\left|\varepsilon^{2(4-p) /(p-2)} \int b\left(\frac{x}{\varepsilon}\right) E_{1}\left(b\left(\frac{x}{\varepsilon}\right)|u|^{2}\right)\right| u\right|^{2} \\
& -\varepsilon_{0}^{2(4-p) /(p-2)} \int b\left(\frac{x}{\varepsilon_{0}}\right) E_{1}\left(b\left(\frac{x}{\varepsilon_{0}}\right)\left|u_{0}\right|^{2}\right)\left|u_{0}\right|^{2} \mid \\
& \leq\left.|\varepsilon|^{2(4-p) /(p-2)}\left|\int b\left(\frac{x}{\varepsilon}\right) E_{1}\left(b\left(\frac{x}{\varepsilon}\right)|u|^{2}\right)\right| u\right|^{2} \\
& -\int b\left(\frac{x}{\varepsilon_{0}}\right) E_{1}\left(b\left(\frac{x}{\varepsilon_{0}}\right)\left|u_{0}\right|^{2}\right)\left|u_{0}\right|^{2} \mid \\
& +\left|\varepsilon^{2(4-p) /(p-2)}-\varepsilon_{0}^{2(4-p) /(p-2)}\right| \\
& \times\left.\left|\int b\left(\frac{x}{\varepsilon_{0}}\right) E_{1}\left(b\left(\frac{x}{\varepsilon_{0}}\right)\left|u_{0}\right|^{2}\right)\right| u_{0}\right|^{2} \mid \\
& :=|\varepsilon|^{2(4-p) /(p-2)} I_{1}+I_{2} \text {. }
\end{aligned}
$$

It is obvious that $I_{2} \rightarrow 0$, as $\varepsilon \rightarrow \varepsilon_{0}$. At the same time, we know

$$
\begin{aligned}
I_{1} \leq & \left.\left|\int\left[b\left(\frac{x}{\varepsilon}\right)-b\left(\frac{x}{\varepsilon_{0}}\right)\right] E_{1}\left(b\left(\frac{x}{\varepsilon}\right)|u|^{2}\right)\right| u\right|^{2} \mid \\
& +\left.\left|\int b\left(\frac{x}{\varepsilon_{0}}\right) E_{1}\left(\left[b\left(\frac{x}{\varepsilon}\right)-b\left(\frac{x}{\varepsilon_{0}}\right)\right]|u|^{2}\right)\right| u\right|^{2} \mid \\
& +\left.\left|\int b\left(\frac{x}{\varepsilon_{0}}\right) E_{1}\left(b\left(\frac{x}{\varepsilon_{0}}\right)\left[|u|^{2}-\left|u_{0}\right|^{2}\right]\right)\right| u\right|^{2} \mid \\
& +\left|\int b\left(\frac{x}{\varepsilon_{0}}\right) E_{1}\left(b\left(\frac{x}{\varepsilon_{0}}\right)\left|u_{0}\right|^{2}\right)\left(|u|^{2}-\left|u_{0}\right|^{2}\right)\right| \\
:= & \Pi_{1}+\Pi_{2}+\Pi_{3}+\Pi_{4} .
\end{aligned}
$$

Estimating the first term $\Pi_{1}$, by Hölder inequality, we know

$$
\begin{aligned}
\Pi_{1}= & \left.\left|\int\left[b\left(\frac{x}{\varepsilon}\right)-b\left(\frac{x}{\varepsilon_{0}}\right)\right] E_{1}\left(b\left(\frac{x}{\varepsilon}\right)|u|^{2}\right)\right| u\right|^{2} \mid \\
\leq & \left(\int\left(\left|b\left(\frac{x}{\varepsilon}\right)-b\left(\frac{x}{\varepsilon_{0}}\right)\right||u|^{2}\right)^{2}\right)^{1 / 2} \\
& \times\left(\int\left|E_{1}\left(b\left(\frac{x}{\varepsilon}\right)|u|^{2}\right)\right|^{2}\right)^{1 / 2} .
\end{aligned}
$$

Since $b(x)$ is bounded and continuous, the operator $E_{1} \epsilon$ $\mathscr{L}\left(L^{2}, L^{2}\right)$, the dominated convergence theorem implies that

$$
\Pi_{1} \longrightarrow 0, \quad \text { as } \varepsilon \longrightarrow \varepsilon_{0} .
$$

Similarly, we can deduce that $\Pi_{2}, \Pi_{3}, \Pi_{4}$ vanishes, as $(\varepsilon, u) \rightarrow$ $\left(\varepsilon_{0}, u_{0}\right)$. Hence

$$
\left|G_{2}(\varepsilon, u)-G_{2}\left(\varepsilon_{0}, u_{0}\right)\right| \longrightarrow 0
$$

as $(\varepsilon, u) \rightarrow\left(\varepsilon_{0}, u_{0}\right)$.

If $(\varepsilon, u) \rightarrow\left(0, u_{0}\right)$, by definition, $G_{2}(0, u)=0$. Since $b(x) \in L^{2}\left(\mathbb{R}^{3}\right)$ is also bounded, we know $b(x) \in L^{6}\left(\mathbb{R}^{3}\right)$, applying Parseval identity and Hölder inequality, we get

$$
\begin{aligned}
4\left|G_{2}(\varepsilon, u)\right| & \leq\left.|\varepsilon|^{2(4-p) /(p-2)} \int\left|b\left(\frac{x}{\varepsilon}\right) E_{1}\left(b\left(\frac{x}{\varepsilon}\right)|u|^{2}\right)\right| u\right|^{2} \mid \\
& \leq|\varepsilon|^{2(4-p) /(p-2)} \int\left|b\left(\frac{x}{\varepsilon}\right)\right|^{2}|u(x)|^{4} \\
& \leq|\varepsilon|^{2(4-p) /(p-2)}\left(\int\left|b\left(\frac{x}{\varepsilon}\right)\right|^{6}\right)^{1 / 3}\left(\int|u|^{6}\right)^{2 / 3},
\end{aligned}
$$

therefore $G_{2}(\varepsilon, u) \rightarrow 0$, as $(\varepsilon, u) \rightarrow(0, u)$. Hence $G=G_{1}+$ $G_{2}$ is continuous and the lemma is proved.

Lemma 8. Under assumptions $\left(a_{1}\right)$ and $\left(b_{1}\right), G^{\prime}$ and $G^{\prime \prime}$ are continuous in $(\varepsilon, u)$.

Proof. $G_{1}^{\prime}$ and $G_{1}^{\prime \prime}$ are continuous in $(\varepsilon, u)$, see [7, Lemma 4.2] for the details. Here we only prove that $G_{2}^{\prime}$ and $G_{2}^{\prime \prime}$ are continuous in $(\varepsilon, u)$.

$$
\text { If }(\varepsilon, u) \rightarrow\left(\varepsilon_{0}, u_{0}\right) \text { with } \varepsilon_{0} \neq 0 \text {, then }
$$

$$
\begin{aligned}
\| G_{2}^{\prime}(\varepsilon, u)- & G_{2}^{\prime}\left(\varepsilon_{0}, u_{0}\right) \| \\
=\sup _{\|v\|=1}\{ & \varepsilon^{2(4-p) /(p-2)} \\
& \times \int b\left(\frac{x}{\varepsilon}\right) E_{1}\left(b\left(\frac{x}{\varepsilon}\right)|u|^{2}\right) u v \\
& -\varepsilon_{0}^{2(4-p) /(p-2)} \\
& \left.\times \int b\left(\frac{x}{\varepsilon_{0}}\right) E_{1}\left(b\left(\frac{x}{\varepsilon_{0}}\right)\left|u_{0}\right|^{2}\right) u_{0} v\right\} \\
\leq \sup _{\|v\|=1}\left\{|\varepsilon|^{2(4-p) /(p-2)}\right. & \\
& \times \int\left(b\left(\frac{x}{\varepsilon}\right) E_{1}\left(b\left(\frac{x}{\varepsilon}\right)|u|^{2}\right) u v\right. \\
& \left.\quad-b\left(\frac{x}{\varepsilon_{0}}\right) E_{1}\left(b\left(\frac{x}{\varepsilon_{0}}\right)\left|u_{0}\right|^{2}\right) u_{0} v\right) \\
& \left.\times\left|\int b\left(\frac{x}{\varepsilon_{0}}\right) E_{1}\left(b\left(\frac{x}{\varepsilon_{0}}\right)\left|u_{0}\right|^{2}\right) u_{0} v\right|\right\} \\
:=|\varepsilon|^{2(4-p) /(p-2)}-\varepsilon_{0}^{2(4-p) /(p-2)} \mid & \sup I_{1}+\sup _{\|v\|=1} I_{2} . \\
\|v\|=1 &
\end{aligned}
$$

Estimating the second term, since $E_{1} \in \mathscr{L}\left(L^{2}, L^{2}\right)$, by Hölder inequality, we know

$$
\begin{aligned}
\sup _{\|v\|=1} I_{2}= & \sup _{\|v\|=1}\left|\varepsilon^{2(4-p) /(p-2)}-\varepsilon_{0}^{2(4-p) /(p-2)}\right| \\
& \times\left|\int b\left(\frac{x}{\varepsilon_{0}}\right) E_{1}\left(b\left(\frac{x}{\varepsilon_{0}}\right)\left|u_{0}\right|^{2}\right) u_{0} v\right|
\end{aligned}
$$




$$
\begin{aligned}
\leq & \sup _{\|v\|=1} C_{0}\left|\varepsilon^{2(4-p) /(p-2)}-\varepsilon_{0}^{2(4-p) /(p-2)}\right| \\
& \times\left(\left.\left.\int\left|b\left(\frac{x}{\varepsilon_{0}}\right)\right| u_{0}\right|^{2}\right|^{2}\right)^{1 / 2}\left(\int\left|b\left(\frac{x}{\varepsilon_{0}}\right) u_{0} v\right|^{2}\right)^{1 / 2} \\
\leq & C_{1}\left|\varepsilon^{2(4-p) /(p-2)}-\varepsilon_{0}^{2(4-p) /(p-2)}\right|\left(\int\left|u_{0}\right|^{6}\right)^{1 / 2} .
\end{aligned}
$$

Thus $\sup _{\|v\|=1} I_{2} \rightarrow 0$ as $\varepsilon \rightarrow \varepsilon_{0}$. Estimating the first term $I_{1}$, we know

$$
\begin{aligned}
& \sup _{\|v\|=1} I_{1}=\sup _{\|v\|=1}\left\{\int\right.\left(b\left(\frac{x}{\varepsilon}\right) E_{1}\left(b\left(\frac{x}{\varepsilon}\right)|u|^{2}\right) u v\right. \\
&\left.\left.-b\left(\frac{x}{\varepsilon_{0}}\right) E_{1}\left(b\left(\frac{x}{\varepsilon_{0}}\right)\left|u_{0}\right|^{2}\right) u_{0} v\right)\right\} \\
& \leq \sup _{\|v\|=1}\left\{\mid \int\right. {\left[b\left(\frac{x}{\varepsilon}\right)-b\left(\frac{x}{\varepsilon_{0}}\right)\right] E_{1}\left(b\left(\frac{x}{\varepsilon}\right)|u|^{2}\right) u v \mid } \\
&+ \mid \int b\left(\frac{x}{\varepsilon_{0}}\right) E_{1} \\
& \times\left(\left[b\left(\frac{x}{\varepsilon}\right)-b\left(\frac{x}{\varepsilon_{0}}\right)\right]|u|^{2}\right) u v \mid \\
&+ \mid \int b\left(\frac{x}{\varepsilon_{0}}\right) E_{1} \\
& \times\left(b\left(\frac{x}{\varepsilon_{0}}\right)\left[|u|^{2}-\left|u_{0}\right|^{2}\right]\right) u v \mid \\
&+ \mid \int b\left(\frac{x}{\varepsilon_{0}}\right) E_{1} \\
&\left.\times\left(b\left(\frac{x}{\varepsilon_{0}}\right)\left|u_{0}\right|^{2}\right)\left(u v-u_{0} v\right) \mid\right\} \\
&:=A_{1}+A_{2}+A_{3}+A_{4} \cdot
\end{aligned}
$$

As in Lemma 7, by Hölder inequality again, we can prove that $A_{i} \rightarrow 0$, as $(\varepsilon, u) \rightarrow\left(\varepsilon_{0}, u_{0}\right), i=1,2,3,4$. Therefore $\left\|G_{2}^{\prime}(\varepsilon, u)-G_{2}^{\prime}\left(\varepsilon_{0}, u_{0}\right)\right\| \rightarrow 0$ as $(\varepsilon, u) \rightarrow\left(\varepsilon_{0}, u_{0}\right)$.

If $\varepsilon_{0}=0$, from the definition of $G_{2}$, we know $\left\|G_{2}^{\prime}\left(0, u_{0}\right)\right\|=$ 0 . Hence

$$
\begin{aligned}
& \left\|G_{2}^{\prime}(\varepsilon, u)\right\| \\
& =\sup _{\|v\|=1}\left|\varepsilon^{2(4-p) /(p-2)} \int b\left(\frac{x}{\varepsilon}\right) E_{1}\left(b\left(\frac{x}{\varepsilon}\right)|u|^{2}\right) u v\right| \\
& \leq \sup _{\|v\|=1}\left\{|\varepsilon|^{2(4-p) /(p-2)}\left(\int\left|b\left(\frac{x}{\varepsilon}\right)\right|^{6}\right)^{1 / 3}\right. \\
& \left.\times\left(\int|u|^{6}\right)^{1 / 2}\left(\int|v|^{6}\right)^{1 / 6}\right\} .
\end{aligned}
$$

And we know $\left\|G_{2}^{\prime}(\varepsilon, u)\right\| \rightarrow 0$, as $\varepsilon \rightarrow 0$. From the above arguments, we know $G^{\prime}=G_{1}^{\prime}+G_{2}^{\prime}$ is continuous in $(\varepsilon, u)$.
In the following we prove that $G^{\prime \prime}$ is continuous in $(\varepsilon, u)$. As we know

$$
\begin{aligned}
G_{2}^{\prime \prime}(\varepsilon, u)[w, v]= & \varepsilon^{2(4-p) /(p-2)} \\
& \times \int\left(b\left(\frac{x}{\varepsilon}\right) E_{1}\left(b\left(\frac{x}{\varepsilon}\right)|u|^{2}\right) w v\right. \\
& \left.+2 b\left(\frac{x}{\varepsilon}\right) E_{1}\left(b\left(\frac{x}{\varepsilon}\right) w u\right) u v\right) .
\end{aligned}
$$

$$
\text { If }(\varepsilon, u) \rightarrow\left(\varepsilon_{0}, u_{0}\right) \text { with } \varepsilon_{0} \neq 0 \text {, then }
$$

$$
\begin{aligned}
& \left\|G_{2}^{\prime \prime}(\varepsilon, u)-G_{2}^{\prime \prime}\left(\varepsilon_{0}, u_{0}\right)\right\| \\
& \quad=\sup _{\|w\|=\|v\|=1}\left|G_{2}^{\prime \prime}(\varepsilon, u)[w, v]-G_{2}^{\prime \prime}\left(\varepsilon_{0}, u_{0}\right)[w, v]\right| \\
& \quad \leq \sup _{\|w\|=\|v\|=1}\left\{I_{1}+I_{2}\right\},
\end{aligned}
$$

where

$$
\begin{aligned}
& I_{1}:=\mid \varepsilon^{2(4-p) /(p-2)} \int b\left(\frac{x}{\varepsilon}\right) E_{1}\left(b\left(\frac{x}{\varepsilon}\right)|u|^{2}\right) w v \\
& \quad-\varepsilon_{0}^{2(4-p) /(p-2)} \int b\left(\frac{x}{\varepsilon_{0}}\right) E_{1}\left(b\left(\frac{x}{\varepsilon_{0}}\right)\left|u_{0}\right|^{2}\right) w v \mid, \\
& I_{2}:=2 \mid \varepsilon^{2(4-p) /(p-2)} \int b\left(\frac{x}{\varepsilon}\right) E_{1}\left(b\left(\frac{x}{\varepsilon}\right) u w\right) u v \\
& \quad-\varepsilon_{0}^{2(4-p) /(p-2)} \int b\left(\frac{x}{\varepsilon_{0}}\right) E_{1}\left(b\left(\frac{x}{\varepsilon_{0}}\right) u_{0} w\right) u_{0} v \mid .
\end{aligned}
$$

We estimate $I_{1}$ only, and $I_{2}$ can be estimated in a similar way, indeed

$$
\begin{aligned}
& I_{1} \leq\left|\varepsilon^{2(4-p) /(p-2)}-\varepsilon_{0}^{2(4-p) /(p-2)}\right| \\
& \quad \times \mid \int b\left(\frac{x}{\varepsilon}\right) E_{1}\left(b\left(\frac{x}{\varepsilon}\right)|u|^{2}\right) w v \\
& \quad-\int b\left(\frac{x}{\varepsilon_{0}}\right) E_{1}\left(b\left(\frac{x}{\varepsilon_{0}}\right)\left|u_{0}\right|^{2}\right) w v \mid \\
& +\left|\varepsilon_{0}\right|^{2(4-p) /(p-2)} \mid \int b\left(\frac{x}{\varepsilon}\right) E_{1}\left(b\left(\frac{x}{\varepsilon}\right)|u|^{2}\right) w v \\
& \quad-\int b\left(\frac{x}{\varepsilon_{0}}\right) E_{1}\left(b\left(\frac{x}{\varepsilon_{0}}\right)\left|u_{0}\right|^{2}\right) w v \mid .
\end{aligned}
$$

Similar to the proof in Lemma 7, we know $I_{1} \rightarrow 0$ as $\varepsilon \rightarrow \varepsilon_{0}$ and $u \rightarrow u_{0}$. Thus we know $\left\|G_{2}^{\prime \prime}(\varepsilon, u)-G_{2}^{\prime \prime}\left(\varepsilon_{0}, u_{0}\right)\right\| \rightarrow 0$ as $(\varepsilon, u) \rightarrow\left(\varepsilon_{0}, u_{0}\right)$. 

know

If $(\varepsilon, u) \rightarrow\left(0, u_{0}\right)$, then from the definition of $G_{2}$, we

$$
\begin{aligned}
& \left\|G_{2}^{\prime \prime}(\varepsilon, u)\right\| \\
& =\sup _{\|w\|=\|v\|=1}\left|G_{2}^{\prime \prime}(\varepsilon, u)[w, v]\right| \\
& \leq \sup _{\|w\|=\|v\|=1}\left\{\varepsilon^{2(4-p) /(p-2)}\right. \\
& \quad \times \int\left(b\left(\frac{x}{\varepsilon}\right) E_{1}\left(b\left(\frac{x}{\varepsilon}\right)|u|^{2}\right) w v\right. \\
& \left.\left.\quad+2 b\left(\frac{x}{\varepsilon}\right) E_{1}\left(b\left(\frac{x}{\varepsilon}\right) w u\right) u v\right)\right\} \\
& :=\sup _{\|w\|=\|v\|=1}\left\{I_{3}+I_{4}\right\} .
\end{aligned}
$$

Using Hölder inequality, we know

$$
\begin{aligned}
I_{3} \leq & C_{0}|\varepsilon|^{2(4-p) /(p-2)}\left(\int\left|b\left(\frac{x}{\varepsilon}\right)\right|^{2}|w v|^{2}\right)^{1 / 2} \\
& \times\left(\int\left|b\left(\frac{x}{\varepsilon}\right)\right|^{2}|u|^{4}\right)^{1 / 2} \\
\leq & C_{0}|\varepsilon|^{2(4-p) /(p-2)}\left(\int\left|b\left(\frac{x}{\varepsilon}\right)\right|^{6}\right)^{1 / 3}\left(\int|u|^{6}\right)^{1 / 3} \\
& \times\left(\int|w|^{6}\right)^{1 / 6}\left(\int|v|^{6}\right)^{1 / 6}, \\
I_{4} \leq & C_{1}|\varepsilon|^{2(4-p) /(p-2)}\left(\int\left|b\left(\frac{x}{\varepsilon}\right)\right|^{6}\right)^{1 / 3}\left(\int|u|^{6}\right)^{1 / 3} \\
& \times\left(\int|w|^{6}\right)^{1 / 6}\left(\int|v|^{6}\right)^{1 / 6} \cdot
\end{aligned}
$$

Therefore

$$
\left\|G_{2}^{\prime \prime}(\varepsilon, u)\right\| \longrightarrow 0 \quad \text { as } \varepsilon \longrightarrow 0 .
$$

From the above arguments, we know $G^{\prime \prime}$ is continuous in $(\varepsilon, u)$ and the proof is complete.

Lemma 9. Assume $\left(a_{1}\right)$ and $\left(b_{1}\right)$ are satisfied. Define

$$
\Gamma(\theta)=-\frac{1}{p} U^{p}(\theta) \int(a(x)-A) .
$$

Then

$$
\begin{aligned}
& \lim _{\varepsilon \rightarrow 0} \frac{G\left(\varepsilon, z_{\theta}\right)}{\varepsilon^{3}}=\Gamma(\theta), \\
& G^{\prime}\left(\varepsilon, z_{\theta}\right)=o\left(\varepsilon^{3 / 2}\right) .
\end{aligned}
$$

Proof. By changing of variable, we know

$$
\begin{aligned}
G_{1}\left(\varepsilon, z_{\theta}\right) & =-\frac{1}{p} \int\left(a\left(\frac{x}{\varepsilon}\right)-A\right) U^{p}(x+\theta) \\
& =-\frac{\varepsilon^{3}}{p} \int(a(x)-A) U^{p}(\varepsilon x+\theta) .
\end{aligned}
$$

Since $a(x)$ is continuous and bounded, the dominated convergence theorem implies that

$$
\lim _{\varepsilon \rightarrow 0} \frac{G_{1}\left(\varepsilon, z_{\theta}\right)}{\varepsilon^{3}}=\Gamma(\theta) .
$$

On the other hand, since $z_{\theta}$ is bounded and $b(x) \in L^{2}\left(\mathbb{R}^{3}\right)$, then, changing of variable, we know

$$
\begin{aligned}
\left|\frac{G_{2}\left(\varepsilon, z_{\theta}\right)}{\varepsilon^{3}}\right|= & \mid \frac{\varepsilon^{2(4-p) /(p-2)-3}}{4} \\
& \quad \times \int b\left(\frac{x}{\varepsilon}\right) E_{1}\left(b\left(\frac{x}{\varepsilon}\right)\left|z_{\theta}\right|^{2}\right)\left|z_{\theta}\right|^{2} \mid \\
\leq & |\varepsilon|^{2(4-p) /(p-2)-3}\left(\int\left|b\left(\frac{x}{\varepsilon}\right)\right|^{2}\left|z_{\theta}\right|^{4}\right) \\
\leq & C_{1}|\varepsilon|^{2(4-p) /(p-2)-3} \int\left|b\left(\frac{x}{\varepsilon}\right)\right|^{2} \\
= & C_{1}|\varepsilon|^{2(4-p) /(p-2)} \int|b(x)|^{2} \longrightarrow 0, \quad \text { as } \varepsilon \longrightarrow 0
\end{aligned}
$$

since $4>p>2$. Thus we obtain

$$
\lim _{\varepsilon \rightarrow 0} \frac{G\left(\varepsilon, z_{\theta}\right)}{\varepsilon^{3}}=\Gamma(\theta) .
$$

Now we are ready to prove

$$
G^{\prime}\left(\varepsilon, z_{\theta}\right)=o\left(\varepsilon^{3 / 2}\right) .
$$

From the proof of [7], we first know

$$
G_{1}^{\prime}\left(\varepsilon, z_{\theta}\right)=o\left(\varepsilon^{3 / 2}\right) \text {. }
$$

Also, since $z$ is bounded, it is easy to check that

$$
\begin{aligned}
\frac{\left\|G_{2}^{\prime}\left(\varepsilon, z_{\theta}\right)\right\|}{|\varepsilon|^{3 / 2}=} & |\varepsilon|^{-3 / 2} \sup _{\|v\|=1}\left|\left(G_{2}^{\prime}\left(\varepsilon, z_{\theta}\right), v\right)\right| \\
= & |\varepsilon|^{2(4-p) /(p-2)-3 / 2} \\
& \times \sup _{\|v\|=1}\left|\int b\left(\frac{x}{\varepsilon}\right) E_{1}\left(b\left(\frac{x}{\varepsilon}\right)\left|z_{\theta}\right|^{2}\right) z_{\theta} v\right| \\
\leq & C_{0}|\varepsilon|^{2(4-p) /(p-2)-3 / 2} \\
& \times \sup _{\|v\|=1}\left\{\left(\left.\int b\left(\frac{x}{\varepsilon}\right)\right|^{2}\left|z_{\theta}\right|^{4}\right)^{1 / 2}\right. \\
& \left.\quad \times\left(\int\left|b\left(\frac{x}{\varepsilon}\right)\right|^{2}\left|z_{\theta}\right|^{2}|v|^{2}\right)^{1 / 2}\right\} \\
\leq & C_{1}|\varepsilon|^{2(4-p) /(p-2)-3 / 2} \\
& \times \sup _{\|v\|=1}\left|\left(\left.\int b\left(\frac{x}{\varepsilon}\right)\right|^{2}\right)^{1 / 2}\left(\int\left|b\left(\frac{x}{\varepsilon}\right) v\right|^{2}\right)^{1 / 2}\right| .
\end{aligned}
$$


Moreover, recall that $b(x) \in L^{2}$ is bounded and use Hölder inequality, we get

$$
\frac{\left\|G_{2}^{\prime}\left(\varepsilon, z_{\theta}\right)\right\|}{|\varepsilon|^{3 / 2}} \leq C_{1}|\varepsilon|^{2(4-p) /(p-2)},
$$

since $4>p$, we have

$$
\lim _{\varepsilon \rightarrow 0} \frac{G_{2}^{\prime}\left(\varepsilon, z_{\theta}\right)}{\varepsilon^{3 / 2}}=0 .
$$

From the above arguments, we know

$$
\lim _{\varepsilon \rightarrow 0} \frac{G^{\prime}\left(\varepsilon, z_{\theta}\right)}{\varepsilon^{3 / 2}}=0,
$$

and the proof is completed.

Proof of Theorem 2. By the exponential decay property of proposition $U$, it is easy to check that $I_{0}^{\prime \prime}$ is a compact perturbation of the identity map, and so it is an index0 Fredholm operator. By Proposition 4, we know that $Z$ is a nondegenerate 3-dimensional critical manifold. From Lemmas 7 to 9, we know all the assumptions of Lemma 5 are satisfied. Since $U$ has a strict (global) maximum at $x=0, \Gamma$ has a strict (global) maximum or minimum at $\theta=0$ depending on the sign of $\int(a(x)-A)$. By the abstract theorem, we know the existence of family solutions $\left\{\left(\varepsilon, u_{\varepsilon}\right)\right\} \subset \mathbb{R} \times H^{1}\left(\mathbb{R}^{3}\right)$. If $2<p<2+4 / 3$, it is easy to check that $\psi_{\varepsilon} \rightarrow 0$ as $\varepsilon \rightarrow 0$.

Remark 10. The hypothesis $\int(a(x)-A) \neq 0$ is used to apply Lemma 5 and has been already used in $[4,7]$. If $\int(a(x)-A)$ is identically zero, we can not conclude that there exist critical points of $I_{\varepsilon}$.

In the following we prove Theorem 3.

Lemma 11. Assume $\left(a_{2}\right)$ and $\left(b_{1}\right)$ are satisfied. Then $G, G^{\prime}$, and $G^{\prime \prime}$ are continuous in $(\varepsilon, u)$.

Proof. Keeping the exponentially decay property of $U$ in mind, the continuity of $G_{1}, G_{1}^{\prime}$, and $G_{1}^{\prime \prime}$ in $(\varepsilon, u)$ can be proved similarly as in [7]. We can also repeat the proof in Lemma 7 to know the continuity of $G_{2}$. Thus the lemma is concluded.

Lemma 12. Assume $\left(a_{2}\right)$ and $\left(b_{1}\right)$ are satisfied. Define

$$
\Gamma(\theta)=-\frac{L}{p+1} \int|x|^{-\gamma} U^{p+1}(x+\theta) .
$$

Then for all $\theta \in R^{3}$, we have

$$
\begin{gathered}
\lim _{\varepsilon \rightarrow 0} \frac{G\left(\varepsilon, z_{\theta}\right)}{\varepsilon^{\gamma}}=\Gamma(\theta), \quad \lim _{|\theta| \rightarrow \infty} \Gamma(\theta)=0, \\
\lim _{\varepsilon \rightarrow 0} \frac{G^{\prime}\left(\varepsilon, z_{\theta}\right)}{\varepsilon^{\gamma / 2}}=0 .
\end{gathered}
$$

Proof. As we know

$$
G_{1}\left(\varepsilon, z_{\theta}\right)=-\frac{\varepsilon^{\gamma}}{p+1} \int\left(a\left(\frac{x}{\varepsilon}\right)-A\right) \frac{|x|^{\gamma}}{\varepsilon^{\gamma}} \frac{U^{p+1}(x+\theta)}{|x|^{\gamma}} .
$$

By assumption $\left(a_{2}\right)$ and the decay property of $U$,

$$
\lim _{\varepsilon \rightarrow 0} \frac{G_{1}\left(\varepsilon, z_{\theta}\right)}{\varepsilon^{\gamma}}=\Gamma(\theta) .
$$

Moreover, by the boundedness of $z_{\theta}$, we know

$$
\begin{aligned}
\left|G_{2}\left(\varepsilon, z_{\theta}\right)\right|= & \frac{\varepsilon^{2(4-p) /(p-2)}}{4} \\
& \times \int b(x) E_{1}\left(b(x)\left|z_{\theta}\right|^{2}\right)\left|z_{\theta}\right|^{2} \\
\leq & \frac{\varepsilon^{2(4-p) /(p-2)}}{4}\left(\left.\left.\int\left|b\left(\frac{x}{\varepsilon}\right)\right| z_{\theta}\right|^{2}\right|^{2}\right) \\
\leq & C_{0}|\varepsilon|^{2(4-p) /(p-2)+3} .
\end{aligned}
$$

Since $3>\gamma$ we obtain

$$
\lim _{\varepsilon \rightarrow 0} \frac{G\left(\varepsilon, z_{\theta}\right)}{\varepsilon^{\gamma}}=\Gamma(\theta) .
$$

To study the property of $G^{\prime}\left(\varepsilon, z_{\theta}\right)$, since $\gamma<3$ and $U$ exponentially decays at infinity, from the proof in [7], we know

$$
\lim _{\varepsilon \rightarrow 0} \frac{G_{1}^{\prime}\left(\varepsilon, z_{\theta}\right)}{\varepsilon^{\gamma / 2}}=0 .
$$

On the other hand, from the boundedness of $Z_{\theta}$ and $b(x)$, we have

$$
\begin{aligned}
\left\|G_{2}^{\prime}\left(\varepsilon, z_{\theta}\right)\right\|= & |\varepsilon|^{2(4-p) /(p-2)} \\
& \times \sup _{\|v\|=1}\left|\int b\left(\frac{x}{\varepsilon}\right) E_{1}\left(b\left(\frac{x}{\varepsilon}\right)\left|z_{\theta}\right|^{2}\right) z_{\theta} v\right| \\
\leq & C_{0}|\varepsilon|^{2(4-p) /(p-2)} \\
& \times \sup _{\|v\|=1}\left\{\left(\left.\int b\left(\frac{x}{\varepsilon}\right)\right|^{2}\left|z_{\theta}\right|^{4}\right)^{1 / 2}\right. \\
& \left.\times\left(\int\left|b\left(\frac{x}{\varepsilon}\right)\right|^{2}\left|z_{\theta}\right|^{2}|v|^{2}\right)^{1 / 2}\right\} \\
\leq & \left.C_{1}|\varepsilon|^{2(4-p) /(p-2)}\right\} \\
& \times \sup _{\|v\|=1}\left|\left(\left.\int b\left(\frac{x}{\varepsilon}\right)\right|^{2}\right)^{1 / 2}\left(\int|v|^{2}\right)^{1 / 2}\right| \\
\leq & C_{2}|\varepsilon|^{2(4-p) /(p-2)+3 / 2} .
\end{aligned}
$$

Since $\gamma<3$, we get

$$
\lim _{\varepsilon \rightarrow 0} \frac{G_{2}^{\prime}\left(\varepsilon, z_{\theta}\right)}{\varepsilon^{\gamma / 2}}=0
$$

From the above arguments, we know

$$
\lim _{\varepsilon \rightarrow 0} \frac{G^{\prime}\left(\varepsilon, z_{\theta}\right)}{\varepsilon^{\gamma / 2}}=0 .
$$


Proof of Theorem 3. From Lemmas 11 and 12, we know that all the assumptions of Lemma 5 are satisfied. Since $\lim _{|\theta| \rightarrow \infty} \Gamma(\theta)=0$ and $\Gamma(0) \neq 0$, we know that there is $R>0$ such that either

$$
\min _{|\theta|=R} \Gamma(\theta)>\Gamma(0) \quad \text { or } \quad \max _{|\theta|=R} \Gamma(\theta)<\Gamma(0) .
$$

By the abstract Theorem 2, we know the existence of family solutions $\left\{\left(\varepsilon, u_{\varepsilon}\right)\right\} \subset \mathbb{R} \times H^{1}\left(\mathbb{R}^{3}\right)$. If $2<p<2+4 / 3$, it is easy to check that $\left(\phi_{\varepsilon}, \psi_{\varepsilon}\right) \rightarrow 0$ as $\varepsilon \rightarrow 0$.

\section{Acknowledgments}

This work is supported by ZJNSF (Y7080008, R6090109, LQ12A01015, Y201016244, 2012C31025), SRPWZ (G20110004), and NSFC (10971194, 11005081, 21207103).

\section{References}

[1] A. Davey and K. Stewartson, "On three-dimensional packets of surface waves," Proceedings of the Royal Society of London A, vol. 338, pp. 101-110, 1974.

[2] R. Cipolatti, "On the existence of standing waves for a DaveyStewartson system," Communications in Partial Differential Equations, vol. 17, no. 5-6, pp. 967-988, 1992.

[3] R. Cipolatti, "On the instability of ground states for a Davey-Stewartson system," Annales de l'Institut Henri Poincaré, Physique Théorique, vol. 58, no. 1, pp. 85-104, 1993.

[4] A. Ambrosetti and M. Badiale, "Homoclinics: PoincaréMelnikov type results via a variational approach," Annales de l'Institut Henri Poincaré: Analyse Non Linéaire, vol. 15, no. 2, pp. 233-252, 1998.

[5] A. Ambrosetti and M. Badiale, "Variational perturbative methods and bifurcation of bound states from the essential spectrum," Proceedings of the Royal Society of Edinburgh A, vol. 128, no. 6, pp. 1131-1161, 1998.

[6] A. Ambrosetti and A. Malchiodi, Perturbation Methods and Semilinear Elliptic Problems on $R^{n}$, vol. 240 of Progress in Mathematics, Birkhäuser, Basel, Switzerland, 2006.

[7] M. Badiale and A. Pomponio, "Bifurcation results for semilinear elliptic problems in $\mathbb{R}^{N}$," Proceedings of the Royal Society of Edinburgh A, vol. 134, no. 1, pp. 11-32, 2004. 


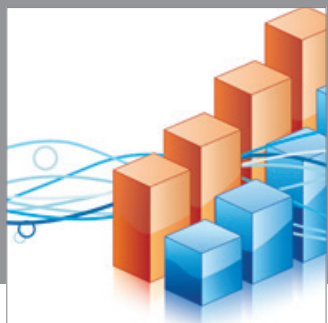

Advances in

Operations Research

mansans

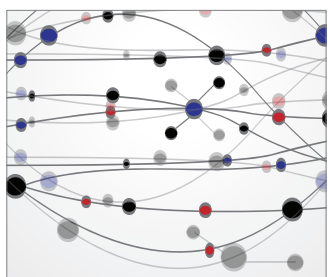

The Scientific World Journal
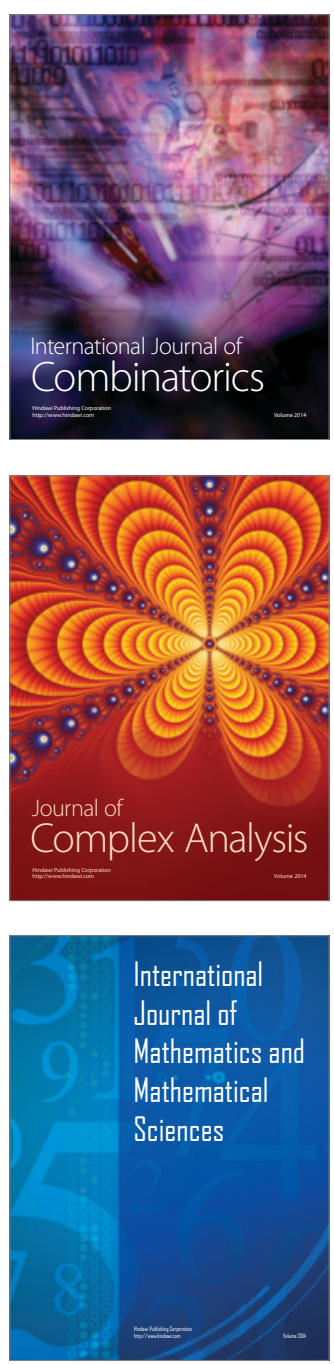
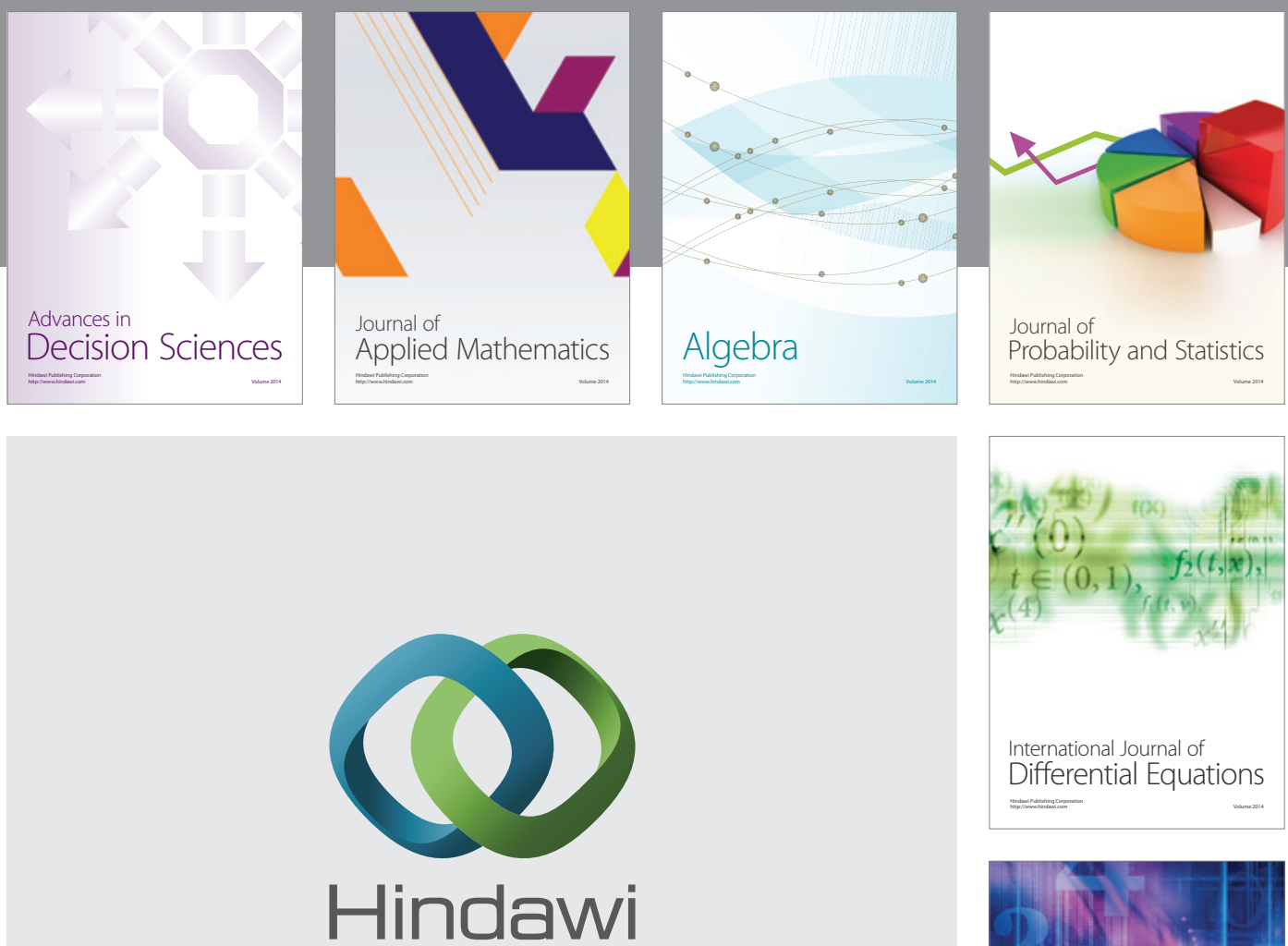

Submit your manuscripts at http://www.hindawi.com
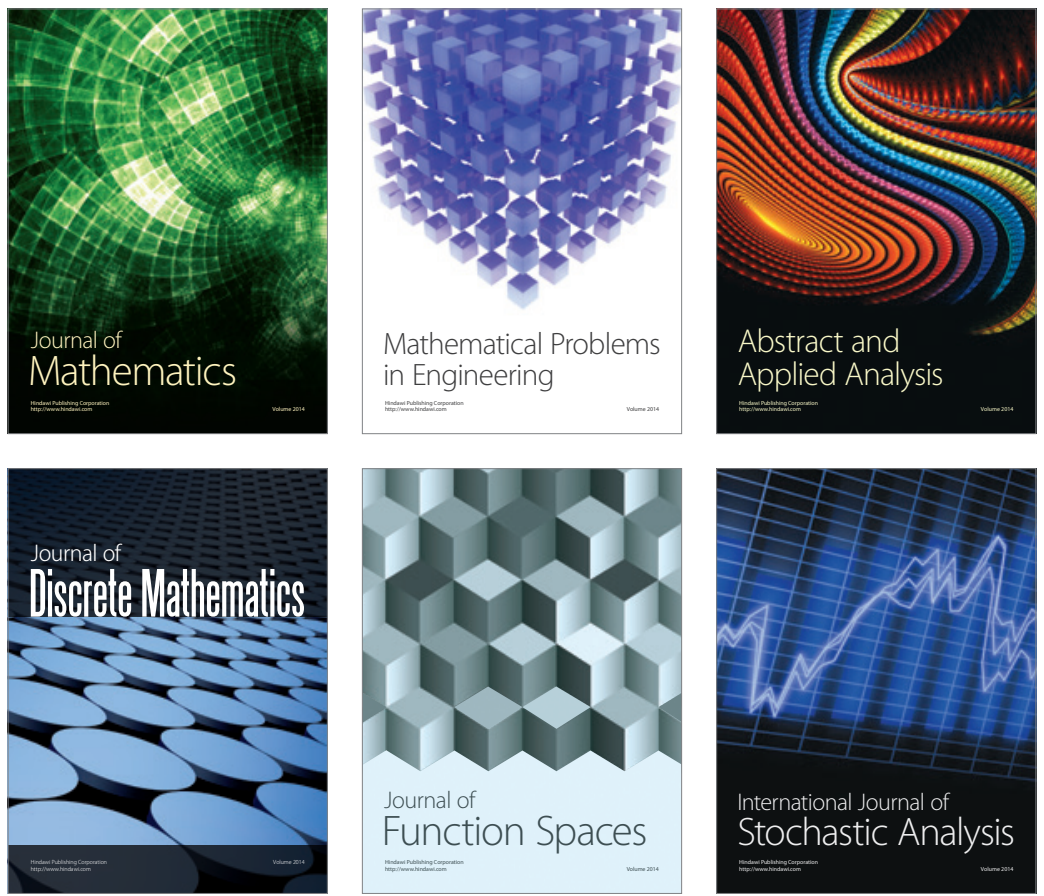

Journal of

Function Spaces

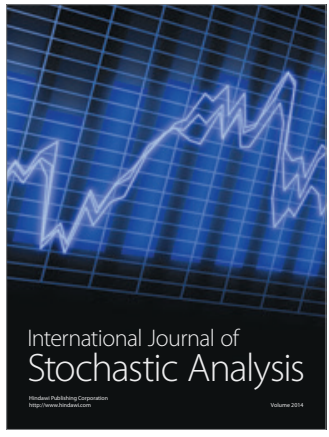

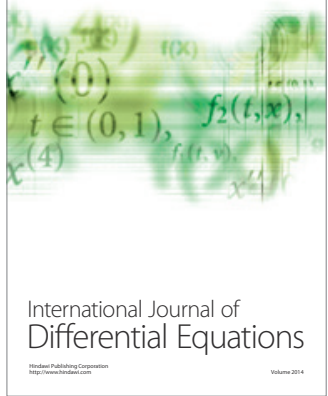
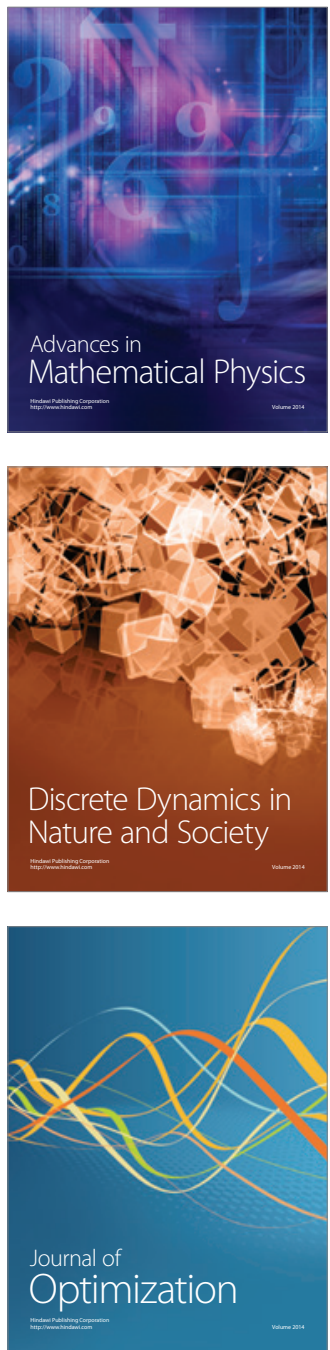